\title{
PLEIADES SYSTEM ARCHITECTURE AND MAIN PERFORMANCES
}

\author{
M. Alain Gleyzes ${ }^{\mathrm{a}, *}$, Lionel Perret ${ }^{\mathrm{a}}$, Philippe Kubik ${ }^{\mathrm{a}}$ \\ ${ }^{a}$ CNES 18, avenue Edouard Belin 31401 Toulouse cedex 4 FRANCE \\ (alain.gleyzes, lionel.perret, philippe.kubik)@cnes.fr
}

Special Session: ISPRS and IAA: Pléiades System and Acquisition Capability

KEY WORDS: Earth observation, system, Pleiades, satellite, ground-segment, architecture, performances

\begin{abstract}
:
France, under the leadership of the French Space Agency (CNES), has set up a cooperative program with Austria, Belgium, Spain, Sweden, in order to develop a space Earth Observation system called PLEIADES.
\end{abstract}

PLEIADES is a dual system, this means that it is intended to fulfill an extended panel of both civilian and Defense user's needs..

This paper reports the status of the satellite after its launch and the in orbit commissioning, the PLEIADES satellite first model has been launched at the end of year 2011, the second model will be launched about 12 months later. It describes the main mission characteristics and performances status. It exposes how the system, satellite and ground segment have been designed in order to be compliant with a dual exploitation between civilian and defense partners.

The system is based on the use of a set of newly European developed technologies to feature the satellite. In order to maximize the agility of the satellite, weight and inertia have been reduced using a compact hexagonal shape for the satellite bus. The optical mission consists in Earth optical observation composed of $0.7 \mathrm{~m}$ nadir resolution for the panchromatic band and $2.8 \mathrm{~m}$ nadir resolution for the four multi-spectral bands. The image swath is about $20 \mathrm{~km}$.

PLEIADES delivers optical high resolution products consisting in a Panchromatic image, into which is merged a four multispectral bands image, orthorectified on a Digital Terrain Model (DTM).

Thanks to the huge satellite agility obtained with control momentum gyros as actuators, the optical system delivers as well instantaneous stereo images, under different stereoscopic conditions and mosaic images, issued from along the track thus enlarging the field of view.

The ground segment is composed of a dual ground center located in CNES Toulouse premises in charge of preparing the dual mission command plan and of the real time contacts with the satellite through a control center. The dual ground center interfaces with several mission centers : one center is built for each Defense partner (France, Spain ...), one center is set up for the Civilian Operator. Each mission center is in charge of managing the programming requests, then, receiving the mission telemetry, processing the data to feed a catalog and an archive, to generate the system products and distribute them to the final user.

System reactivity has been optimized with a chronology based on three mission planning activations per day. The mission plan is uploaded to the satellite just before flyby over East Asia, Europe and North America. In addition a Direct Tasking mode is available for commercial Image Receiving Stations.

\section{INTRODUCTION}

\subsection{Pleiades mission overview}

The Pleiades project was undertaken, under the leadership of the French Space Agency (CNES), as part of an intergovernmental agreement setting out the objectives and general principles of cooperation between France and Italy in the field of Earth observation. Signed on 29 January 2001 in Turin, the agreement makes particular provision for the building of a dual-purpose observation system, ORFEO, with submetric resolution, including an optical component, Pleiades, developed by France and a radar component, Cosmo-SkyMed, developed by Italy.
Cooperation agreements relating to Pleiades have also been signed with Austria, Belgium, Spain and Sweden. In return, they have been granted a right to programming requests and access to the system archive, proportional to their contribution to development according to schemes similar to those used for the Spot family.

The Pleiades system is a truly dual-purpose optical observation system. As a complement to the French military space observation system Helios 2 and the Spot satellites, it has been designed to meet the needs of both Defense and civilian users, institutional and also commercial.

The purpose of Pleiades is to deliver optical images of submetric resolution to these two categories of customers, while 
meeting the stringent requirements of each one: those of Defense in terms of priority (50 high priority images will be allocated each day) and confidentiality of requests, and those of civilian users in terms of acquisition capability and coverage. The Dual System is specified to fulfill a broad spectrum of applications, in the field of cartography, agriculture and forestry, geology and hydrology, marine applications, Earth science, resource management, land use, law enforcement and risk management according to scientific, institutional and commercial customers

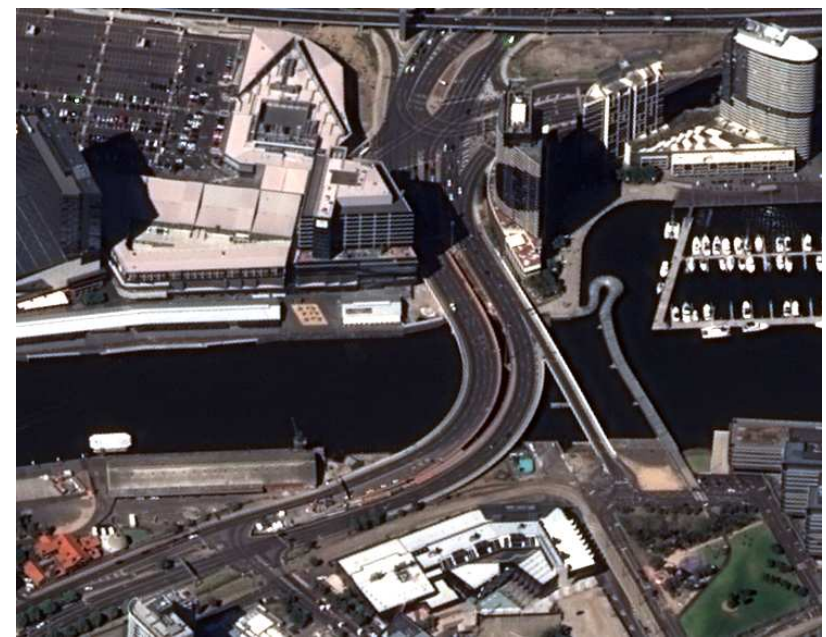

Figure 1: Pleiades 1A image of Melbourne acquired during commissioning (C) CNES Copyright 2012

Given the dual nature of this system, two types of access are defined to schedule the satellites' tasks:

- The Defense channel used by Ministry of Defense beneficiaries for High Priority Defense programming requests.

- The Civil channel used by civilian users. It will be operated by a civilian Operator.

The civilian data distribution is delegated to Astrium GeoInformation Services (ex Spot Image) through a Public Service Delegation : $40 \%$ of the resources of the system will be reserved for institutional users of the cooperative countries for non commercial activities. CNES has granted an exclusive license to the Civilian Operator allowing him to process, distribute and commercialize the data and products on the worldwide market.

\subsection{Key mission performances}

The Pleiades system consists of a constellation of two optical satellites (visible and near-infrared range) each weighing a ton, positioned on a quasi-circular, Sun-synchronous orbit of 695 $\mathrm{km}$ altitude, at a local hour at descending node of 10:30 am.

The first Pleiades satellite was launched by a Soyuz rocket on the night of 16 to 17 December 2011, from the European spaceport at Kourou in French Guiana. The second satellite will be launched about one year later.

Worldwide coverage and a daily accessibility to any point on the globe are requested, and fulfilled by the use of both satellites simultaneously in orbit and $180^{\circ}$ phase shifted. This concept is necessary for two reasons:

- To ensure the accessibility and prompt imaging required by Defense and Civil Security missions.

- To ensure the coverage capability required for mapping and land planning needs.
The Pleiades system offers:

- daily access to any point on the globe (with its two satellites)

- a panchromatic channel with a $70 \mathrm{~cm}$ vertical viewing resolution.

- four spectral bands (blue, green, red and near-infrared) with a $2.8 \mathrm{~m}$ resolution.

- field of view of $20 \mathrm{~km}$ in vertical viewing.

- acquisition capacity, in a single pass, of a 100x100 km² mosaic of images.

- virtually instantaneous acquisition capacity for stereoscopic pairs (and even triplets) of $20 \mathrm{~km}$ up to $300 \mathrm{~km}$.

- cloud-free image coverage of $2,500,000 \mathrm{~km}^{2}$ per year.

In addition it will offer very precise image location $(<12$ meters without ground control points) enabling optimal use of data in Geographic Information Systems (GISs).

\section{SATELLITE DESCRIPTION}

CNES entrusted the building of the two satellites to EADS Astrium (satellite prime contractor) and Thales Alenia Space (high-resolution instrument prime contractor).

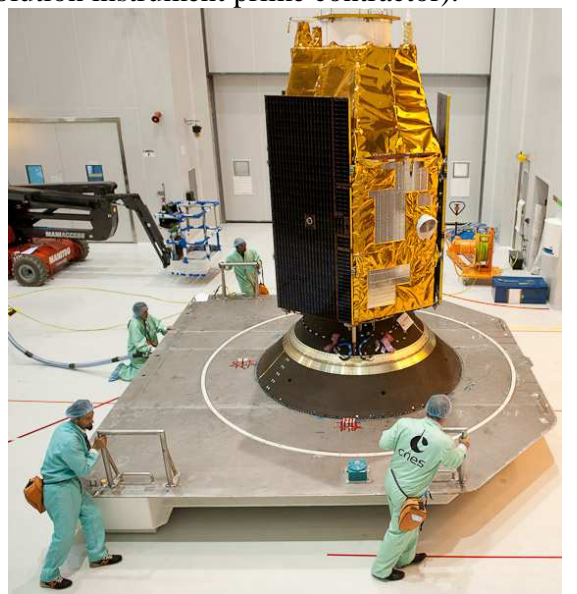

Figure 2: Pleiades 1A a few days before launch

\subsection{Architecture}

Pleiades'agility is a key factor enabling it to provide the service expected by users.

The satellite is able to off-point rapidly around its axis (yaw, pitch, roll). This high agility is made possible by a very compact design, achieved by: (Fig 3)

- fixed, rigid solar panels.

- equipment built into the satellite to reduce the vessel's inertia. - the high-resolution instrument partly embedded inside the bus.

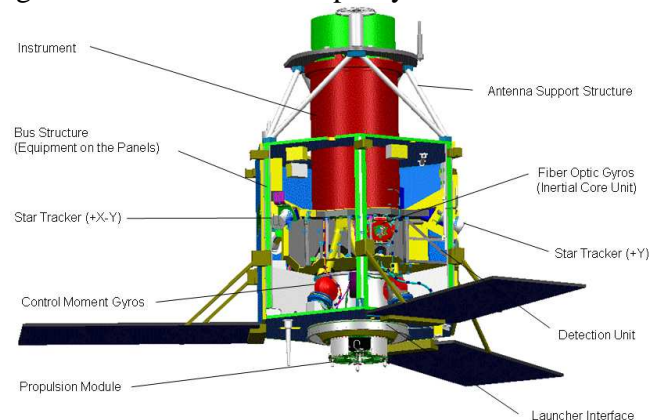

Figure 3: satellite architecture

- a sophisticated control mechanism thanks to a new generation of control moment gyros.(Fig 4) 


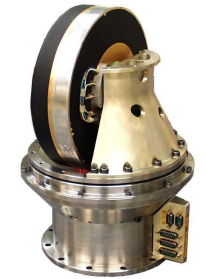

Figure 4: 15 Nms CMG actuator

The attitude control system uses 4 fiber-optic gyroscopes and 3 star trackers to provide attitude accuracy compatible with the system location specification of the products. The orbit navigation is performed by an autonomous navigator using the DORIS system.

The Pleiades satellites have been designed for a theoretical lifetime of 5 years. However, propellant and other consumables have been sized for more than 7 years and the example of the Spot and Helios satellites shows that this period may well be exceeded in orbit.

\subsection{Payload}

The instrument (Fig 5) is based on a design concept with very high dimensional stability combining a carbon/carbon structure with Zerodur ${ }^{\circledR}$ mirrors. For optimal in-flight performance, the instrument includes an innovative thermal refocusing device which avoids the need for a complex mechanism. (Fig 6)

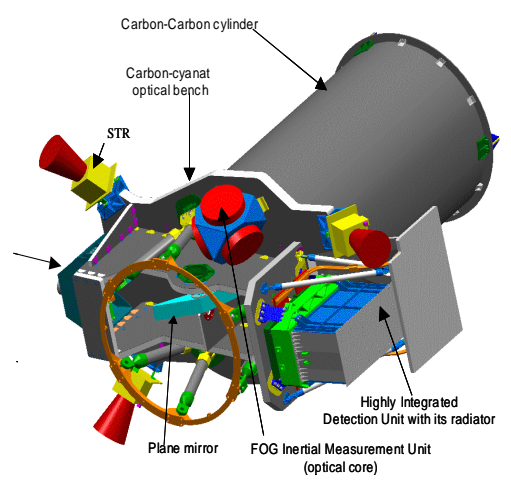

Figure 5: Instrument Configuration

\section{Optical Architecture}

The optical solution chosen for the telescope is a Korsch type combination, with a primary mirror size of $650 \mathrm{~mm}$ diameter and a focal length of $13 \mathrm{~m}$. (Fig 6)

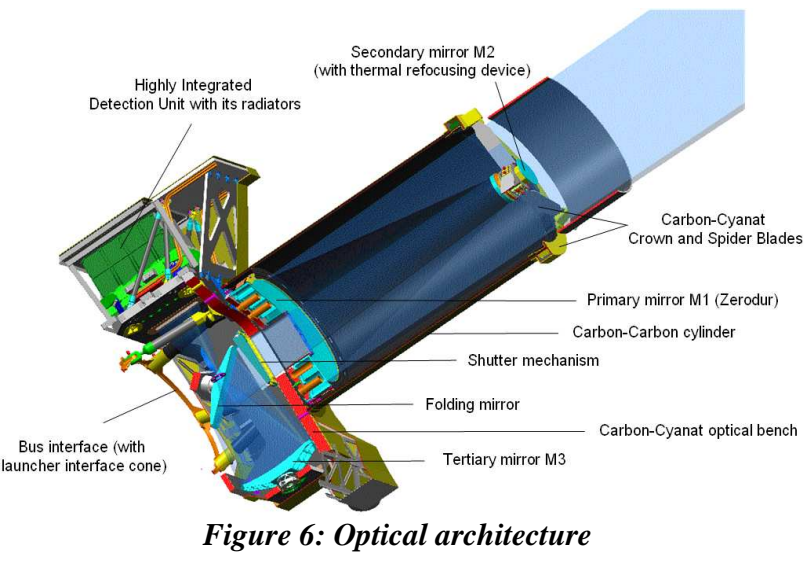

This multi-spectral, high-resolution $(70 \mathrm{~cm})$ and large-field $(20$ $\mathrm{km}$ ) optical instrument, weighing $200 \mathrm{~kg}$, produces images in the visible and near-infrared ranges

\section{Detection}

Back-thinned TDI CCD image sensors (Fig 7) are used for panchromatic detection channel, with a maximum of 20 integration lines. They can be used thanks to an optimized guidance strategy of the satellite line of sight, micro-vibrations levels minimization, specific geometrical accommodation of detector lines in the focal plane and telescope optical distortion minimization.

These devices are back-illuminated for high quantum efficiency and sensitivity. Their TDI format enables them to capture high resolution images without slowing down the satellite. They offer 6,000 active pixels per line and a pixel size and pitch of $13 \mu \mathrm{m}$ square. An architecture with anti-blooming structure in each pixel ensures that image outputs are not jeopardized (smeared) by specular reflections.

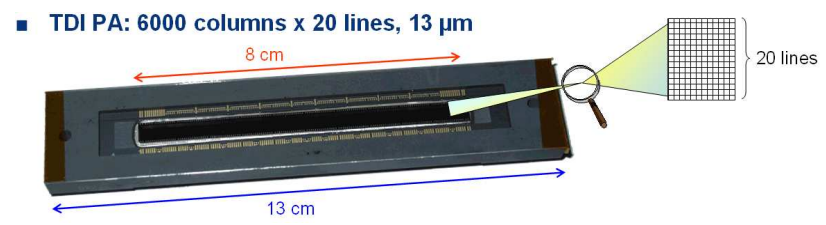

Figure 7: panchromatic TDI sensor

The multi-spectral detection channel is realized with 5 sensors of 1500 pixels per line each, with a pixel size of $52 \mu \mathrm{m}$ (Fig 8). Each sensor consists in a four lines assembly, enabling four colors imaging (blue, green, red, near infrared). Interferometric filters directly stuck down on the detector glass window provide coloring of these four channels.

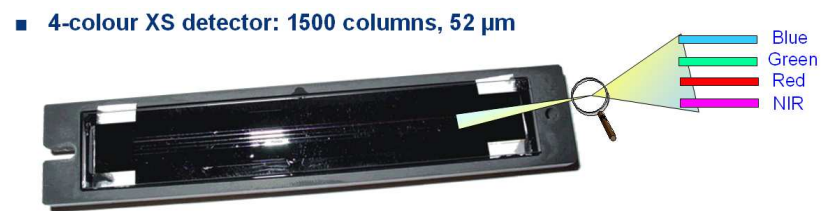

Figure 8: multi-spectral sensor

Five sensors of each kind are used simultaneously to obtain a wide field of view (about $20 \mathrm{~km}$ ). (Fig 9)

Image reconstruction at the level of the inter-array areas is one of the tasks of the ground segment.[1]

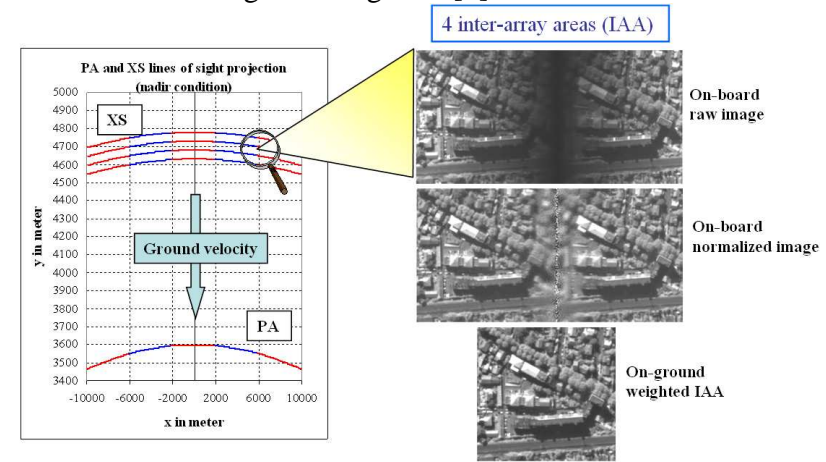

Figure 9: image reconstruction

\section{Image chain}

The video data are compressed on-board thanks to the Payload Data Compression Unit and then transmitted to the ground with a $4.5 \mathrm{Gbits} / \mathrm{s}$ output rate. A wavelet transform algorithm is used, enabling compression rates at 2.8 bits per pixel. 
The compressed data are then memorized in the Solid State Mass Memory. This memory has a storage capacity of 600 Gbits at the end of life.

The data are then encrypted and coded following a trellis-coded scheme in 8-PSK type modulators coupled to Traveling Waves Tube power Amplifiers (TWTA). They are multiplexed and down-linked with an omni-directional $64^{\circ}$ aperture horn antenna. The output rate is nominally of $465 \mathrm{Mbits} / \mathrm{s}$, on three individual channels of $155 \mathrm{Mbits} / \mathrm{s}$ each.

\section{MAIN MISSION CHARACTERISTICS}

\subsection{Agility, Acquisition capabilities and revisit time}

\section{Agility}

Due to its high agility and high performance of the guidance algorithms, the satellite is capable of targetting image along any ground direction within $47^{\circ}$ of vertical viewing position, with very low maneuvers durations between two consecutive images.

This high agility permits :

- to ensure a good reactivity of the system in order to satisfy the urgent needs of the defense or the civil securities

- to minimize scheduling conflicts within the dual use framework, and therefore better meet the simultaneous needs of all users

- to acquire image in any direction which will allow, for example, coastlines or river routes to be monitored, which could be very useful for optimizing programming in a crisis case (flooding, tsunami, etc.).

- to enlarge the swath by taking in the same pass adjacent stripes. Mosaic images ranging from $60 \times 200 \mathrm{~km}^{2}$ (3 strips) to $120 \times 65 \mathrm{~km}^{2}$ (6 strips) with $20^{\circ}$ access authorized, and from $60 \times 340 \mathrm{~km}^{2}$ (3 strips) to $140 \times 105 \mathrm{~km}^{2}$ (6 strips) with $30^{\circ}$ access authorized, can be acquired.

- to acquire in the same pass stereoscopic pairs or tri stereoscopic triplets even with low base over height ratio, a very important improvement in order to avoid hidden objects in urban area (occlusions). Figure 10 shows the maximum length of stereoscopic coverage acquired from the same orbit with several $\mathrm{B} / \mathrm{H}$, either for stereo pairs or for triplets.

\begin{tabular}{|c|c|c|}
\hline B/H & Stereo length & Tri-stereo length \\
\hline 0.1 & $\mathbf{2 5} \mathbf{~ k m}$ & - \\
0.2 & $\mathbf{8 0 ~} \mathbf{~ k m}$ & $\mathbf{2 5} \mathbf{~ k m}$ \\
0.4 & $\mathbf{1 9 5} \mathbf{~ k m}$ & $\mathbf{8 0 ~} \mathbf{~ m}$ \\
0.6 & $\mathbf{3 1 5} \mathbf{~ k m}$ & $\mathbf{1 3 5} \mathbf{~ k m}$ \\
0.8 & $\mathbf{3 5 0} \mathbf{~ k m}$ & $\mathbf{2 0 1} \mathbf{~ k m}$ \\
1 & $\mathbf{3 5 0} \mathbf{~ k m}$ & $\mathbf{2 6 0} \mathbf{~ k m}$ \\
\hline
\end{tabular}

Figure 10: Maximum length of stereo coverage

- To perform calibrations on stars or moon images or using "exotics" guidance methods.[2], .[4], .[5], .[7],

During a single overpass of an area of $1000 \times 1000 \mathrm{~km}^{2}$, each Pleiades satellite acquires about 20 targets with metric resolution (fig 11). In a smaller theatre of operations (100x200 $\left.\mathrm{km}^{2}\right), 16$ targets may be acquired.

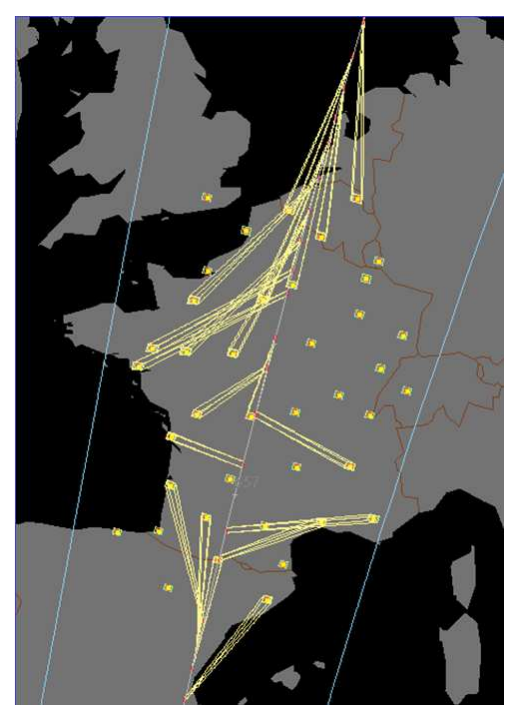

Figure 11: 20 targets on a 1000x1000 $\mathrm{km}^{2}$ area

This amazing agility has been fully demonstrated during the in flight commissioning phase. Five days after launch, the control momentum gyros torque has been unleashed to acquire a set of images with very strong relative pointing angles. The angular rate of the satellite has reached values up to $3.4 \% \mathrm{sec}$ to perform the maneuvers between two data captures :

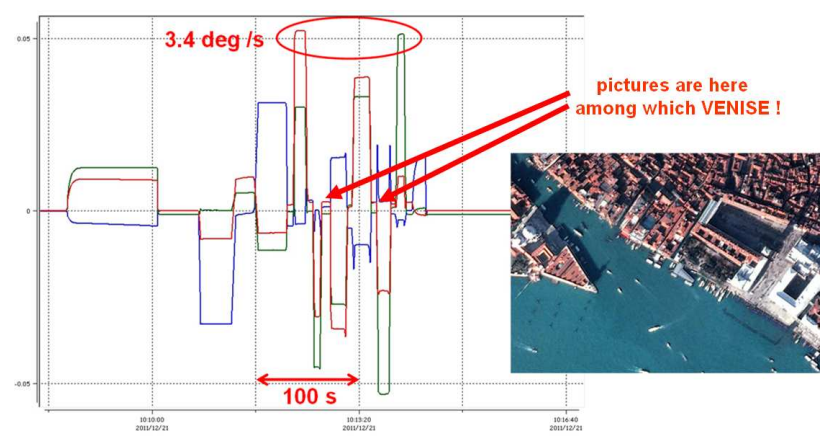

Figure 12: 3 axis angular rate / time sec

In addition to stereoscopic acquisitions, during commissioning, Pleiades agility has been pushed to its limits in order to acquire more than 30 images in a single pass to produce a sequence of animated images allowing to highlight human activities on the targeted area.(Fig 13)

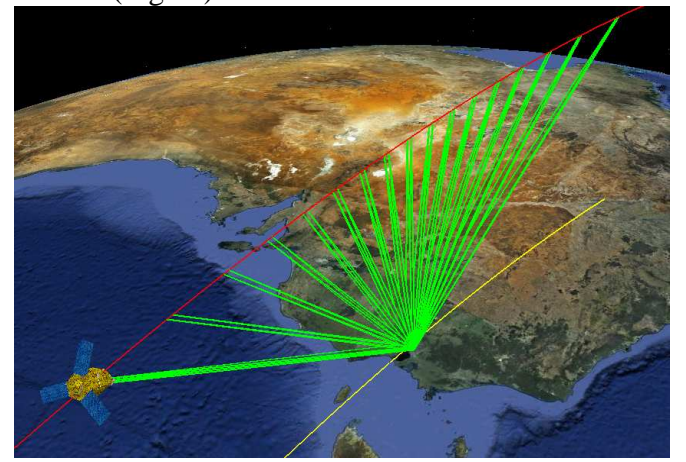

Figure 13: example of video acquisition mode over Melbourne

\section{Revisit time}

This high acquisition capability is accompanied by a revisit interval of less than 24 hours to meet both civil and military requirements 
The orbit has been selected in order to minimize the revisit time with either one or two satellites in orbit. A one-satellite system needs a viewing angle of $47^{\circ}$ to access to any location on the globe in 2 days, whereas with 2 satellites, a viewing angle of $43^{\circ}$ is sufficient to access everywhere daily. The revisit time for different viewing angles is given hereafter:

\begin{tabular}{|c|c|c|}
\hline Viewing angle & 1 satellite & 2 satellites \\
\hline $5^{\circ}$ & 26 days & 13 days \\
\hline $20^{\circ}$ & 7 days & 5 days \\
\hline $30^{\circ}$ & 5 days & 4 days \\
\hline
\end{tabular}

Figure 14: revisit time

\subsection{System chronology and reactivity}

\section{Routine chronology}

The system chronology has been adapted in order to optimize reactivity. Three mission plans are computed per day and uplinked to each satellite

- Morning plan ( 6:00 UTC): Optimization over Europe Africa. Uplink is performed over the Kerguelen station

- Afternoon plan ( 13:00 UTC): Optimization over America. Uplink is performed over the Kiruna station

- Evening plan ( 23:00 UTC): Optimization over Asia. Uplink is performed on the ascending part of the orbit using Toulouse station.

The ground segment is able to take into account last-minute customer request up to 2 hours before the satellite pass over the ground station and to tune the mission plan according to the most recent weather forecast.

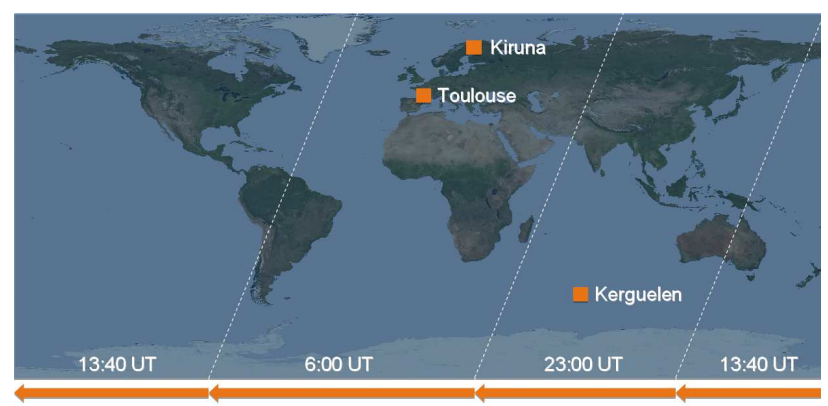

Figure 15: System chronology

\section{Direct Tasking}

In parallel to this routine chronology, Pleiades system offers a Direct Tasking mode to dedicated regional receiving stations equipped with $\mathrm{S}$ and $\mathrm{X}$ bands capabilities.

This mode offers the best reactivity from collection planning to mission ready data, to support ultimate near-real time applications. Direct tasking process follows the hereafter sequence of events: (Fig 15)

- an orbit slot is booked up to 24 hours before the satellite pass.

- the customer's collection plan is prepared and validated. It can be refined and optimized up to 30 minutes before acquisition (considering the latest weather conditions or urgent requests).

- the tasking plan is compiled into commands plan, then ciphered and uploaded.
- the satellite acquires and downloads simultaneously the image data to the Direct tasking station, guarantying a near-real time service.

- the data is automatically produced : 30 minutes from reception to usable information.

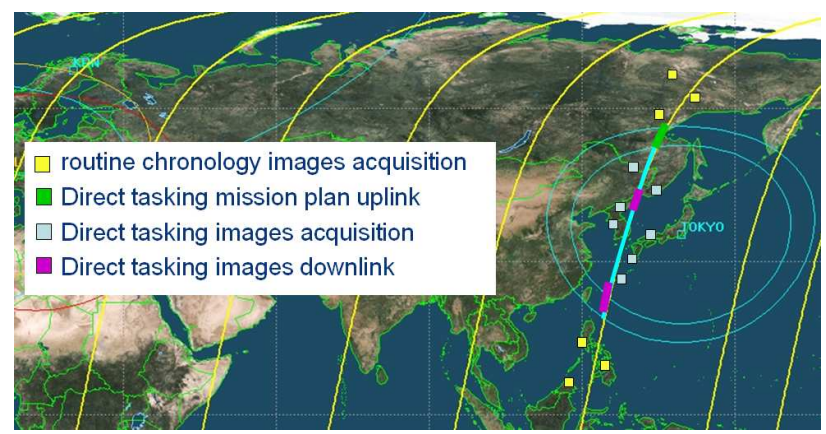

Figure 16: Direct tasking

\subsection{System products}

System Image product levels based on data collected by Pleiades satellites include : [1]

- $\quad$ Raw image: 5 strips panchromatic and multi spectral images, this product will remain internal to the system and is not distributed

- $\quad$ Perfect sensor product: this product is a raw image taken by a perfect sensor with the following characteristics

- Straight (no distortion) and regularly sampled PA/XS lines of sight

- Perfectly registered PA and XS retina

- System-level MTF for PA

- Polynomial attitude fitting the mean estimated attitude

- Estimated actual orbital ephemeris and perfect time stamping

this product is intended to be used for value added processing which can be performed with simplified interfaces

- Ortho images: image with PANCHROMATIC and B0,B1,B2,B3 re-sampled in mapped ground geometry and system geometrical model corrections (standard geometrically corrected product). Corrections coming from global existing DTM (SPOT5 HRS instrument $10 \mathrm{~m}$ accuracy DTM for example) is applied.

- Mosaic products: in the case of lateral multi-band mode acquisition, the system will supply all the orthoimages acquired in a georeferenced common geometry stitched together to generate a single product.

Endogenous high precision DTM (around $1.5 \mathrm{~m}$ accuracy) can be extracted from the Pleiades stereo pairs or triplet. [9] This product is foreseen as a future extension of the system but is not available in the first release of the ground segment.

For levels Perfect sensor, Ortho images, and Mosaic products, true and false colors Pan-Sharpened Multispectral images are available. These products are resampled at $0.5 \mathrm{~m}$ in order to preserve all the frequential information acquired by the instrument and to make the products robust to further ground processing.

\section{GROUND SEGMENT OVERVIEW}

The ground segments were entrusted to CapGemini and CS-SI (programming system), Thales services (image system) and a consortium involving EADS Astrium, Cap Gemini, CS-SI and Thales services (integration of user centers), while INDRA has 
been given responsibility for the Spanish Defense mission centre. The receiving stations have been developed by Zodiac.

\section{1 architecture}

While CNES is prime contractor for the ground segment, its various components are located at different sites and managed by different operators. (Fig 17)

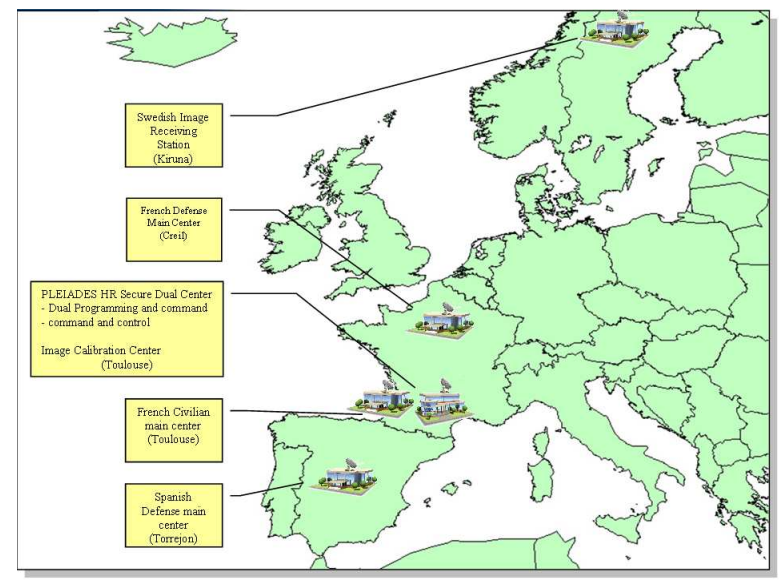

Figure 17: ground centers locations

- The dual control centre is being hosted by CNES in Toulouse. Its role is to:

- schedule all programming requests, by gathering them from Civil channel and Defense channel users,

- manage the satellites on a daily basis, receiving telemetry for verifying its good health, and uploading all the commands enabling it to fulfill its mission,

- calibrate the satellites and their instruments, for the benefit of all users. The Image calibration Center is in charge of computing and distributing the image processing parameters obtained during instrument calibration operations.

The mission centre of the civilian operator, Astrium GeoInformation Services (ex Spot Image) in Toulouse, will satisfy all the demands of institutional users from partner countries as well as commercial users. Its role is to:

- accept and manage user requests,

- generate the requested work plan and send it to the dual control centre three times a day,

- receive, archive and process image telemetry,

- manage a catalogue of images.

A set of Regional Image Receiving Stations deployed under the responsibility of the PHR commercial Operator, will allow local reception and processing of PLEIADES data all over the world. The first one is already installed in Sweden near Kiruna. Its polar location allows to get frequent contacts with the satellite to empty the content of the on board mass memory.

- The French and Spanish defense mission centers (located at Creil near Paris and Torrejón near Madrid, respectively) serve each country's Defense users. Their basic functions are similar to those of the civilian operator's centre, with some differences relating to interfaces and security.

\section{CONCLUSIONS}

Launched on December $17^{\text {th }} 2011$, Pleiades 1 A high resolution optical satellite delivered its first image 3 days after. It has been thoroughly tested and validated during the commissioning phase led by CNES.

Four months after launch, even if some of them cannot be accurately measured before end of June, the main image quality performances have been assessed and appear to be very good. Details on the performances can be found in [2], [3], [4], [5], [6], [7], [8] and [9].

Pleiades has already acquired more than 10000 images, the system is very robust and the satellite availability is excellent. The second satellite, whose launch is planned end of this year, will complete the constellation and make it available to the users.

Success of Pleiades is now in the hands of operational teams that will focus their energies to satisfy the users needs and keep Pleiades in good health during several years!

\section{ACKNOWLEDGEMENTS}

The author would like to thank all the partners, European and French institutional for having trusted CNES to develop this Pleiades program. Special thanks go to CNES hierarchy who has followed and encouraged the project team in the objective to promote technical innovation in European space industry. Final acknowledgements are reserved to the teams, CNES as well as industrial, involved in the Pleiades project development that made this success possible.

\section{REFERENCES}

[1] C. Panem \& al, "Pleiades HR system products performances after in orbit commissioning phase", ISPRS Melbourne 2012.

[2] L Lebegue \& al, "PLEIADES-HR image quality commissioning", ISPRS Melbourne 2012.

[3] S. Lachérade \& al, "Pleiades absolute calibration : first results", ISPRS Melbourne 2012.

[4] S. Fourest \& al, "Star-based methods for Pleiades HR commissioning", ISPRS Melbourne 2012.

[5] G. Blanchet \& al, "Pleiades-HR Innovative Techniques for Radiometric Image Quality Commissioning", ISPRS Melbourne 2012.

[6] C. Latry \& al, "Restoration technique for Pleiades-HR panchromatic images", ISPRS Melbourne 2012.

[7] D. Greslou \& al, "Pleiades-HR innovative techniques for Geometric Image Quality Commissioning”, ISPRS Melbourne 2012.

[8] F. deLussy \& al, "Pleiades HR in flight geometrical calibration : Localisation and mapping of the focal plane", ISPRS Melbourne 2012.

[9] JM. Delvit \& al, “Attitude assessment using Pléiades-HR capabilities”, ISPRS Melbourne 2012. 